\title{
Dorsolateral subthalamic neuronal activity enhanced by median nerve stimulation characterizes Parkinson's disease during deep brain stimulation with general anesthesia
}

\author{
Sheng-Tzung Tsai, MD, ${ }^{1,3}$ Wei-Yi Chuang, PhD, ${ }^{5}$ Chung-Chih Kuo, PhD, ${ }^{4}$ Paul C. P. Chao, PhD, ${ }^{5}$ \\ Tsung-Ying Chen, MD, ${ }^{2,3}$ Hsiang-Yi Hung, MD, ${ }^{1}$ and Shin-Yuan Chen, MD, MS ${ }^{1,3}$

\begin{abstract}
${ }^{1}$ Departments of Neurosurgery and ${ }^{2}$ Anesthesiology, Buddhist Tzu Chi General Hospital, Hualien; ${ }^{3}$ Departments of Medicine and ${ }^{4}$ Physiology, Tzu Chi University, Hualien; and ${ }^{5}$ Department of Electrical Engineering, National Chiao-Tung University, Hsinchu, Taiwan
\end{abstract}

\begin{abstract}
OBJECT Deep brain stimulation (DBS) surgery under general anesthesia is an alternative option for patients with Parkinson's disease (PD). However, few studies are available that report whether neuronal firing can be accurately recorded during this condition. In this study the authors attempted to characterize the neuronal activity of the subthalamic nucleus (STN) and elucidate the influence of general anesthetics on neurons during DBS surgery in patients with PD. The benefit of median nerve stimulation (MNS) for localization of the dorsolateral subterritory of the STN, which is involved in sensorimotor function, was explored.
\end{abstract}

METHODS Eight patients with PD were anesthetized with desflurane and underwent contralateral MNS at the wrist during microelectrode recording of the STN. The authors analyzed the spiking patterns and power spectral density (PSD) of the background activity along each penetration track and determined the spatial correlation to the target location, estimated using standard neurophysiological procedures.

RESULTS The dorsolateral STN spiking pattern showed a more prominent bursting pattern without MNS and more oscillation with MNS. In terms of the neural oscillation of the background activity, beta-band oscillation dominated within the sensorimotor STN and showed significantly more PSD during MNS $(p<0.05)$.

CONCLUSIONS Neuronal firing within the STN could be accurately identified and differentiated when patients with PD received general anesthetics. Median nerve stimulation can enhance the neural activity in beta-band oscillations, which can be used as an index to ensure optimal electrode placement via successfully tracked dorsolateral STN topography.

http://thejns.org/doi/abs/10.3171/2014.11.JNS141208

KEY WORDS deep brain stimulation; general anesthesia; median nerve stimulation; microelectrode recording; subthalamic nucleus; functional neurosurgery

S UBTHALAMIC nucleus (STN) deep brain stimulation (DBS) has been shown to be an effective treatment for advanced Parkinson's disease (PD) and yields good long-term motor outcome. ${ }^{25}$ Conventionally, STNDBS is a surgical procedure conducted while the patient receives a local anesthetic and remains awake to ensure accurate electrophysiological mapping. In addition, this approach allows physicians to conduct intraoperative macrostimulation tests for motor response while minimizing potential side effects of stimulation. However, if a patient with PD is experiencing drastic off-medication symptoms, such as anxiety, painful dystonia, and respiratory distress, the patient may be precluded from this lengthy surgical procedure..$^{16,26} \mathrm{We}$ previously reported our long-term results describing patients with PD who underwent STN-DBS under conditioned general anesthe-

ABBREVIATIONS DBS = deep brain stimulation; ISI = interspike interval; LFP = local field potential; $M A C=$ minimal alveolar concentration; MER = microelectrode recording; MNS = median nerve stimulation; PD = Parkinson's disease; PSD = power spectral density; SSEP = somatosensory evoked potential; $S T N=$ subthalamic nucleus; UPDRS = Unified Parkinson's Disease Rating Scale. 
sia with desflurane. ${ }^{14}$ Microelectrode recording (MER) is possible when patients are deeply sedated, such as during general anesthesia. ${ }^{10}$

Microelectrode mapping during STN-DBS is an essential procedure to optimize the placement of DBS electrodes along the STN. ${ }^{19}$ The somatotopic organization of the STN was determined using MER, which showed that the movement-related cells that control the arm were located laterally and at the rostral and caudal poles of the STN, whereas the cells that control leg movement were located medially and centrally. It is crucial to implant the DBS electrode into this motor subregion to ensure a good outcome. ${ }^{20}$ Although somatotopic mapping within the STN during MER may facilitate optimal placement of the DBS electrodes, the associated shortcomings of this process are that it is time consuming and requires the patient to be awake during the procedure in most hospitals. In our previous study, we showed the feasibility of conducting MER during conditioned general anesthesia under desflurane, which allowed a good long-term outcome. ${ }^{14}$ However, the topography of the STN may not be delineated by MER signals due to partial anesthetic suppression. This characteristic may lead to a higher stimulation side effect during clinical follow-up. ${ }^{5}$ In addition to determining the correlation between the STN topography and the characteristic signal, enhancing MER neuronal signals under conditioned general anesthesia might facilitate the improvement of DBS outcomes.

The pathological and characteristic neuronal oscillations of the STN have been revealed in animal models of PD and human patients. ${ }^{17}$ Beta-band (13-30 Hz) oscillations are not only distinct to the dorsolateral STN and able to be used to predict DBS electrode placement, but can also be manipulated by a patient's active movement.,27 Placing the stimulating electrode on "target" is the crucial factor for a favorable outcome. In addition to movementrelated change of STN firing, previous studies have also tried to localize the target area of the basal ganglia nuclei and STN using somatosensory evoked potentials (SSEPs) elicited by median nerve stimulation (MNS). However, SSEPs coupled with MNS failed to demonstrate the characteristic changes on STN regions, and these studies used macroelectrode recordings. ${ }^{12,18}$ Furthermore, neurophysiological recordings have been widely employed recently to investigate the status of cortical neurons and altered levels of unconsciousness under general anesthesia. ${ }^{6}$ By examining conditioned general anesthesia during surgery, this protocol may allow us to study the extent to which general anesthesia can modulate neuronal firing in the STN. The application of MNS may also explain the mechanisms by which peripheral nerve stimulation may facilitate or hinder CNS oscillation.

\section{Methods \\ Patient Selection}

Eight consecutive patients with PD who underwent bilateral STN-DBS in the Hualien Tzu Chi General Hospital from January 2010 to November 2012 were enrolled in this study (Table 1). All patients met the United Kingdom PD Brain Bank diagnostic criteria, with at least 2 of the cardinal symptoms present. Before surgery, each patient underwent a levodopa test to confirm a positive levodopa response ( $>28 \%$ improvement in the Unified Parkinson's Disease Rating Scale [UPDRS] Part III score). Brain MRI was performed preoperatively to rule out structural abnormalities in each patient. All medications were withdrawn at least 12 hours before surgery. Before surgery in the medication "off" condition, the mean Hoehn and Yahr score was $3.0 \pm 0.46$ (range 2.5-4) and the mean UPDRSIII score was $41.1 \pm 10.3$ (range 27-57). The Institutional Review Board at Tzu Chi General Hospital approved the surgical and evaluation procedures. Informed consent was obtained from each patient.

\section{Imaging and Targeting}

Images were obtained using a 1.5-T MRI unit (General Electric). The standard settings included T1-weighted axial images of 0.75 -mm thickness and T2-weighted axial images of 2-mm thickness. Each sequence was performed in contiguous slices. The images were transferred to the DICOM database using the Picture Archiving and Communication System and the stealth neuronavigation workstation (Medtronic). The image fusion software fused the two sets of MR images to form a 3D reconstruction. The tentative surgical target coordinates for the tip of the permanent implantable electrode were set at the central, lowest border of the STN by direct visualization on MRI, as previously described in detail. ${ }^{4}$ A Leksell G-frame unit (Elekta Instrument, Inc.) was used for the stereotactic procedure. The patient was resting in a straight supine position, and the head frame was secured in a Mayfield adaptor. The target coordinates were applied to the stereotactic frame and the working stage.

\section{Anesthetic Procedure}

All patients received a general anesthetic with endotracheal intubation. Anesthesia was induced initially by administering regular narcotic agents. All patients were maintained using desflurane inhalation during the surgical procedures. The depth of the anesthesia was maintained at 0.5-1.0 minimal alveolar concentration (MAC), and each patient's heart rate and blood pressure were monitored to ensure that the patient avoided a cough reflex or any change in heart rate and blood pressure during the MER procedure. ${ }^{14}$

\section{Electrical Stimulation of the Median Nerve}

A Digitimer constant current stimulator (model DS7A) was used to apply electrical stimulation to the contralateral median nerve. A stimulation electrode was placed on the wrist (cathode, median nerve $2 \mathrm{~cm}$ proximal to the wrist crease; anode, $2 \mathrm{~cm}$ distal to the cathode), and the stimulation parameters included a pulse width of $0.2 \mu \mathrm{sec}$, an intensity of $30 \mathrm{~mA}$, and a frequency of $33 \mathrm{~Hz}$.

\section{Microelectrode Recording Procedure}

The MER device is 10-40 $\mu \mathrm{m}$ in diameter and measures $200 \mathrm{~mm}$ in length, with a 10 -mm-long bare tungsten tip (FHC). The recording impedance was usually between 0.5 and $1 \mathrm{M} \Omega$. The microelectrode was mounted on a 
TABLE 1. Clinical characteristics of the 8 patients with PD

\begin{tabular}{lcccccc}
\hline Case No. & $\begin{array}{c}\text { Age of Onset } \\
\text { (yrs), Sex }\end{array}$ & $\begin{array}{c}\text { Disease } \\
\text { Duration (yrs) }\end{array}$ & $\begin{array}{c}\text { Hoehn \& Yahr } \\
\text { Stage (off) }\end{array}$ & \multicolumn{2}{c}{ Levodopa Response in UPDRS Part III } \\
\hline 1 & $53, \mathrm{M}$ & 7 & 2.5 & 38 & 27 & $\%^{*}$ \\
\hline 2 & $44, \mathrm{~F}$ & 12 & 3 & 45 & 21 & 53.3 \\
\hline 3 & $59, \mathrm{M}$ & 13 & 3 & 32 & 22 & 31.2 \\
\hline 4 & $52, \mathrm{~F}$ & 8 & 3 & 57 & 20 & 64.9 \\
\hline 5 & $52, \mathrm{~F}$ & 10 & 3 & 39 & 12 & 69.2 \\
\hline 6 & $37, \mathrm{M}$ & 8 & 4 & 54 & 29 & 46.3 \\
\hline 7 & $53, \mathrm{M}$ & 11 & 3 & 27 & 16 & 40.7 \\
\hline 8 & $48, \mathrm{~F}$ & 8 & 2.5 & 37 & 13 & 64.9 \\
\hline Mean $\pm \mathrm{SD}$ & $49.8 \pm 6.7$ & $9.6 \pm 2.2$ & $3.0 \pm 0.5$ & $41.1 \pm 10.3$ & $20.0 \pm 6.1$ & $50.0 \pm 15.7$ \\
\hline
\end{tabular}

* Calculated by: drug off - drug on/drug off.

microdriver and then placed in the STN. The signal obtained from the tip of the microelectrode was transmitted to the intraoperative MER system (Leadpoint, Medtronic), where the signal was amplified $(\times 10)$, filtered $(300 \mathrm{~Hz}-3$ $\mathrm{kHz}$ ), and monitored acoustically using a Leadpoint device. Recording started $10 \mathrm{~mm}$ above the planned target coordinates. The dorsal border of the STN was defined by the appearance of multiunit activity among the neurons, which was also accompanied by increased background activity. We advanced the microelectrode from a distance of $200 \mu \mathrm{m}$ to $500 \mu \mathrm{m}$ to detect the robust firing of neurons. The discharge from each neuron was recorded for 10 seconds. After we identified and recorded individual typical STN neuronal firing along the STN path, we activated the median nerve of the contralateral limb and recorded another 10 seconds of interstimulation STN firing. Each recording was saved to the Leadpoint system for offline analysis. The ventral border of the STN was identified by the absence of typical STN firing. In accordance with recently published literature, we characterized the subterritories of the STN into 3 regions, which were separated along the stereotactic z-axis. We defined the upper-third subregion of the STN as the dorsolateral part, and all recorded neuronal firing activities within this area were exported for MER analysis.

The microelectrode typically passed through the thalamus, the zona incerta, the STN, and the underlying substantia nigra along a trajectory to the STN. Passive movement of the contralateral limbs was tested during MER in the STN to determine whether any movement-related neuronal firing changes occurred. Neurons were regarded as movement related if there was an audible alteration in the neuronal discharge that was reproducible and synchronous with the passive movement. ${ }^{1}$ The selection of the final trajectory for electrode implantation depended on the length of the STN neuronal firing and on the presence of arm and/or leg passive movements related to the activity of the STN neuron. We did not perform any macrostimulation tests after the electrodes (Medtronic 3387 DBS leads) were permanently implanted.

\section{Localization of Active Contact With Postoperative CT}

Consecutive axial CT slices of the brain with a thick- ness of $1.25 \mathrm{~mm}$ were obtained after surgery to exclude intracranial complications and to localize the postoperative electrode coordinates through image fusion with the preoperative MRI ${ }^{24}$ An acute stimulation test was performed 1 week after surgery to select the optimal stimulation contact and parameters for chronic stimulation.

\section{MER Signal Offline Analysis Processing}

The MER data of 112 single STN units were obtained from 8 patients. We then obtained the MERs of the dorsolateral STN neurons. The analysis criteria for MERs included spike detection, spike pattern differentiation, neural background activity extraction, and power spectral density (PSD) estimation.

\section{Spike Detection}

Neuronal activity recordings of the dorsolateral STN were exported offline to the Spike 2 software suite (Version 5, Cambridge Electronic Device). In addition, the sampling rate of all MERs was $24 \mathrm{kHz}$. Spikes were differentiated for spike detection using threshold detection and template matching. Identified single units were verified using principle component analysis and visual inspection (Fig. 1). However, setting the proper threshold was critical for spike detection.

Typically, the MER signal consists of the spiking activity of most nearby neuronal units and background electrical activity due to several sources, including more remotely located units, measurement noise, and various artifacts. ${ }^{11}$ In this study, we assumed the input signal $\left(\mathrm{x}_{(\mathrm{t})}\right)$ to be a linear combination of transient action potential signals $\left(\mathrm{x}_{\text {ap }}\right)$ and band-limited Gaussian noise $\left(\mathrm{x}_{\mathrm{n}}\right)$, which is shown as follows:

$$
\mathrm{x}_{(\mathrm{t})}=\mathrm{x}_{\mathrm{ap}}+\mathrm{x}_{\mathrm{n}}
$$

Moreover, we assumed that the Gaussian background noise had a mean of zero. ${ }^{3}$ In other words, the noise can be fully described by its root mean square value, which is equivalent to its standard deviation $\sigma$. In this study, the detection threshold was set at 3 times the estimated background root mean square. ${ }^{3,9}$ When the waveform amplitude exceeded the threshold, we searched for the signal 


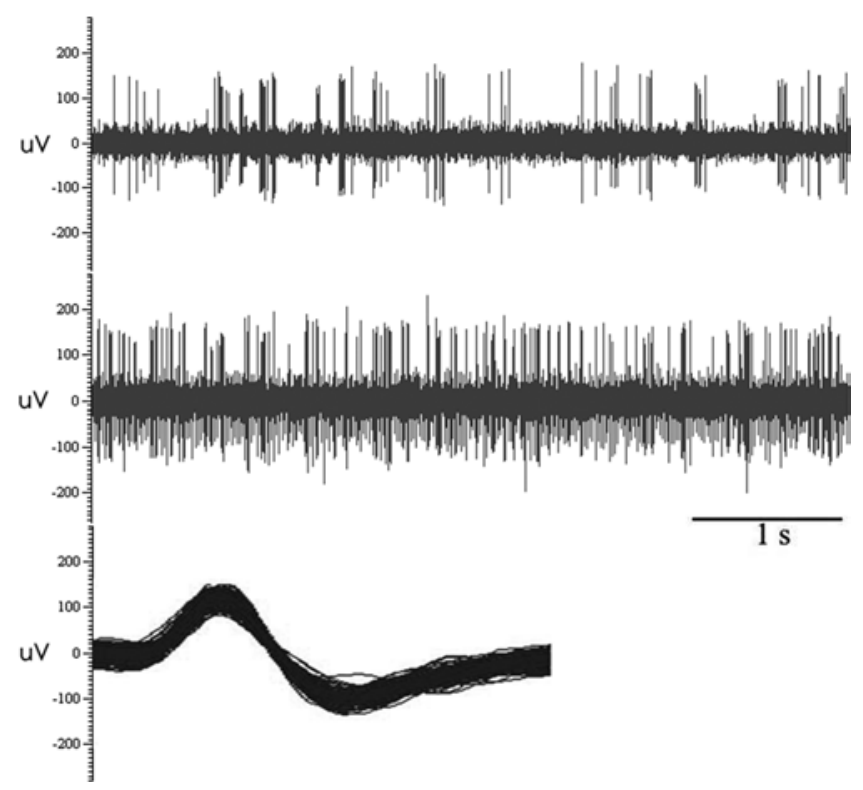

FIG. 1. Various firing patterns from single-unit recordings for 5 seconds between MNS off (upper) and MNS on (center). Both MER traces were recorded from the same STN location. Spike waveforms of STN neurons after template matching (lower) are also shown.

wave for a distinct spike. The threshold setting was calculated using Matlab (MathWorks).

\section{Spike Pattern Differentiation}

The spike times of identified single units were imported into Neuroexplorer (Version 4, Nex Technologies) to determine the mean firing rates, interspike intervals (ISIs), and autocorrelograms. The asymmetry index and the coefficient of variance of the ISI were calculated. Autocorrelograms of the single-unit firing patterns were characterized into 3 categories: 1) irregular, which exhibited an initial trough that rose smoothly to a steady state; 2 ) bursty, which was characterized by an initial peak followed by a decay to a steady state; and 3) oscillatory, in which discharges with similar refractory periods exhibited repetitive neuronal firing with multiple equidistant peaks and troughs (Fig. 2). ${ }^{22}$

\section{Neural Background Activity Extraction and PSD Estimation}

Background activity extraction is an essential step while examining the neuronal background activities of the dorsolateral STN evoked by MNS. In this process, the traces in the segments from $0.5 \mu \mathrm{sec}$ before to $2.5 \mu \mathrm{sec}$ after each spike timestamp were replaced by a random spike-free 3-usec consecutive signal from a random location within the same trace. ${ }^{17}$ Moreover, to explore the oscillatory characteristics of the background activity, we determined the PSD of beta-band activity $(13-30 \mathrm{~Hz})$. The PSD was estimated using Welch's method. The signal was divided equally into segments with $50 \%$ overlap. Each section was windowed with Hamming windows whose length was equal to the number of samples in 2 seconds.

\section{Statistical Analysis}

Numerical values of clinical outcomes measured before and after the operation were compared using paired t-tests. The firing rate, asymmetry index, and coefficient of variance of each ISI were compared between the MSN on and off conditions using paired t-tests. The proportions of various firing patterns with MNS either on or off were compared using chi-square tests; $\mathrm{p}$ values $<0.05$ were considered statistically significant.

\section{Results}

\section{Clinical Outcome After STN-DBS and Localization}

Postoperative DBS substantially improved the clinical status of the patients. A follow-up examination at 3 months after surgery revealed that STN-DBS significantly improved UPDRS-III scores by $38.68 \%$ (med off and DBS off, $38.0 \pm 3.61$; med on and DBS on, $23.3 \pm 5.86$; $<0.001)$. The mean stimulation parameters of channel 1 (left STN-DBS electrode)/channel 2 (right STN-DBS electrode) were $3.23 \pm 0.23 \mathrm{~V}$ and $2.91 \pm 0.70 \mathrm{~V}$, respectively; 60-usec (both channels) pulse width; and frequencies of $122.5 \pm 13.89 \mathrm{~Hz}$ and $118.75 \pm 15.53 \mathrm{~Hz}$, respectively.

\section{Analysis of Single Dorsolateral STN Unit Activity}

We performed a mean of $57.13 \pm 9.19$ MNS stimulations along the STN path. The mean number of MER trajectories was $1.33 \pm 0.82$ per patient side and the recorded length of the STN was $4.69 \pm 0.82 \mathrm{~mm}$. The mean firing frequencies for 10-second intervals of the MNS off and on conditions were $34.40 \pm 26.16 \mathrm{~Hz}(\mathrm{n}=46)$ and 30.85 $\pm 20.25 \mathrm{~Hz}(\mathrm{n}=66)$, respectively, which were not significantly different $(\mathrm{p}>0.05$, t-test). The asymmetry index and coefficient of variance of the ISIs before and after MNS were similar (Table 2). Based on the autocorrelogram analysis, the most frequently observed activity pattern of all dorsolateral STN units with MNS off was bursty (19/46 units, 41.3\%), followed by irregular (16/46 units, $34.7 \%$ ) and oscillatory (11/46 units, 24.0\%) firing patterns. Moreover, the observed activity patterns of all dorsolateral STN units with MNS on were oscillatory (31/66 units, $47.0 \%$ ), followed by bursty (19/66 units, 28.8\%) and irregular (16/66 units, 24.2\%) firing patterns. Oscillatory neuronal activity was found with a significantly increased incidence between MNS off and on (odds ratio 2.82, p < 0.05 , chi-square test). In contrast, the bursty firing patterns were significantly decreased in MNS on relative to MNS off (odds ratio 0.57, $\mathrm{p}<0.05$, chi-square test).

\section{Analysis of Background Oscillation}

Oscillatory activity of the neuronal firing pattern is correlated with the synchronous background activity of the MER data. To explore the background oscillation, we estimated the spectrum character (i.e., the PSD) of the background activity while neuronal spikes were detected. In addition, the PSD of dorsolateral subthalamic neurons in the beta band exhibits significant features. In this study, the PSD of background activity in the beta band was examined to explore the oscillation evoked by MNS. Both the PSD of each patient and that of the whole group in the 
A
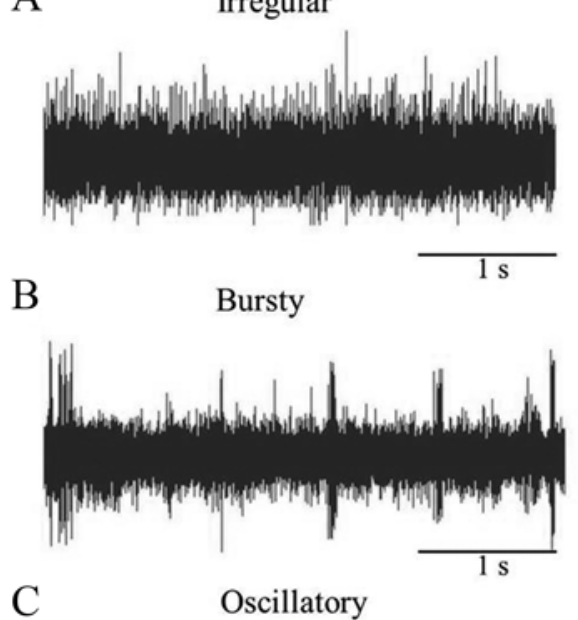

$\mathrm{C}$

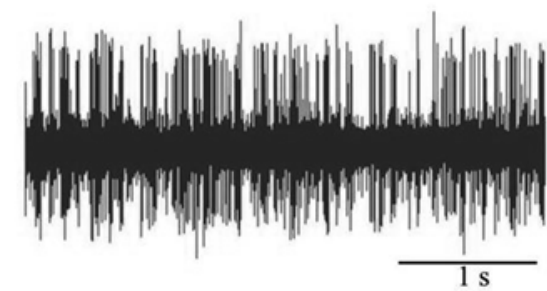

$\mathrm{D}$

E
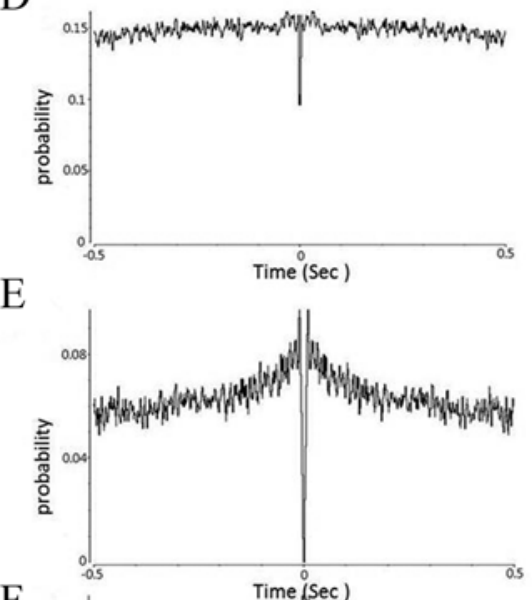

F

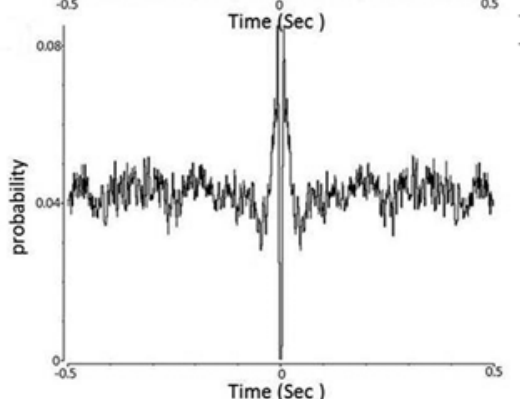

G

$\mathrm{H}$
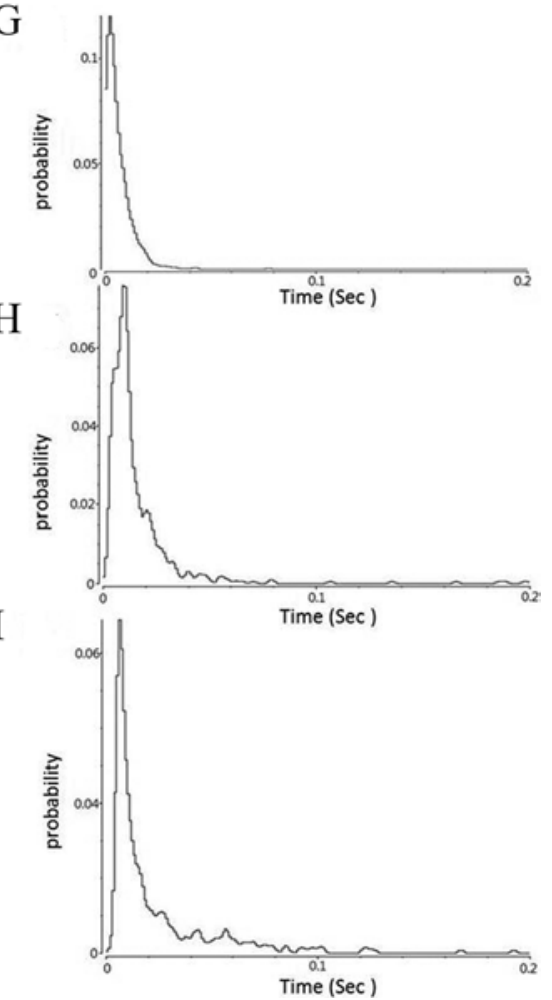

FIG. 2. Raw recordings (A-C), autocorrelograms (D-F), and ISI histograms (G-I) of 3 types of spiking patterns after spike sorting and postprocessing in the dorsolateral STN. Shown are typical examples of an irregular activity pattern (A, D, and G), a bursty firing pattern (B, E, and $\mathrm{H})$, and an oscillatory firing pattern (C, F, and I).

beta band were significantly increased while MNS was on compared with while MNS was off (Table 3; t-test).

\section{Discussion}

After we analyzed the physiological features of the STN from single neuron recordings in patients with PD, we demonstrated that STN neurons could be recorded and identified while patients were under general anesthesia. In addition to discriminating the characteristics among 3 types of STN firing while a patient was under general anesthesia, we could identify pattern shifts of STN neuronal firing after peripheral nerve stimulation using MNS.

A clear distinction of the anatomical boundaries among the 3 subregions (motor, associative, and limbic areas) of the STN is difficult to achieve in human subjects. ${ }^{15}$ Through strict mapping of the MER axis and preoperative MRI, we determined the characteristics of the dorsolateral and motor subterritory neurons of the STN in patients with PD under general anesthesia, which were mostly in

TABLE 2. The firing frequency, as well as the coefficient of variance and asymmetry index of the ISI, within the dorsolateral STN

\begin{tabular}{|c|c|c|c|c|c|c|}
\hline \multirow[b]{2}{*}{ MNS } & \multirow[b]{2}{*}{ Spike Pattern } & \multirow{2}{*}{$\begin{array}{l}\text { No. of } \\
\text { Neurons }\end{array}$} & \multirow[b]{2}{*}{$\%$} & \multirow{2}{*}{$\begin{array}{c}\text { Firing } \\
\text { Frequency }(\mathrm{Hz})\end{array}$} & \multicolumn{2}{|c|}{$\mid \mathrm{ISI}$} \\
\hline & & & & & Coefficient of Variance & Asymmetry index \\
\hline \multicolumn{7}{|l|}{ Off } \\
\hline & Irregular & 16 & 34.7 & $58.28 \pm 29.41$ & $1.04 \pm 0.19$ & $0.34 \pm 0.15$ \\
\hline & Bursty & 19 & 41.3 & $25.43 \pm 12.21$ & $1.36 \pm 0.40$ & $0.25 \pm 0.12$ \\
\hline & Oscillatory & 11 & 24.0 & $16.37 \pm 8.42$ & $1.33 \pm 0.21$ & $0.13 \pm 0.04$ \\
\hline & Total & 46 & 100 & $34.40 \pm 26.16$ & $1.22 \pm 0.33$ & $0.25 \pm 0.15$ \\
\hline \multicolumn{7}{|l|}{ On } \\
\hline & Irregular & 16 & 24.2 & $42.69 \pm 26.97$ & $0.87 \pm 0.19$ & $0.50 \pm 0.32$ \\
\hline & Bursty & 19 & 28.8 & $36.77 \pm 20.43$ & $1.26 \pm 0.33$ & $0.24 \pm 0.08$ \\
\hline & Oscillatory & 31 & 47.0 & $21.11 \pm 9.06$ & $1.27 \pm 0.35$ & $0.16 \pm 0.06$ \\
\hline & Total & 66 & 100 & $30.85 \pm 20.25$ & $1.17 \pm 0.36$ & $0.27 \pm 0.22$ \\
\hline
\end{tabular}


TABLE 3. The PSD distribution of background activity in the beta band

\begin{tabular}{lcc}
\hline & \multicolumn{2}{c}{ Mean PSD \pm SD (arbitrary units) } \\
\cline { 2 - 3 } Case No. & MNS Off & MNS On \\
\hline 1 & $0.0020 \pm 0.0007$ & $0.0024 \pm 0.0005^{*}$ \\
\hline 3 & $0.0024 \pm 0.0006$ & $0.0034 \pm 0.0011 \dagger$ \\
\hline 4 & $0.0033 \pm 0.0021$ & $0.0051 \pm 0.0021^{*}$ \\
\hline 2 & $0.0030 \pm 0.0022$ & $0.0063 \pm 0.0009 \dagger$ \\
\hline 5 & $0.0023 \pm 0.0006$ & $0.0059 \pm 0.0013 \dagger$ \\
\hline 6 & $0.0019 \pm 0.0007$ & $0.0042 \pm 0.0007 \dagger$ \\
\hline 7 & $0.0054 \pm 0.0015$ & $0.0062 \pm 0.0013^{*}$ \\
\hline 8 & $0.0010 \pm 0.0003$ & $0.0020 \pm 0.0003 \dagger$ \\
\hline Average & $0.0027 \pm 0.0004$ & $0.0044 \pm 0.0004 \dagger$ \\
\hline
\end{tabular}

$* p<0.05$.

$+p<0.001$

agreement with previously published results in terms of the asymmetry index and coefficient of variance of the ISIs. It is interesting that lower mean firing frequencies $(34.4 \mathrm{~Hz})$ were recorded in patients under general anesthesia compared with patients undergoing DBS with a local anesthetic $(41.2-58 \mathrm{~Hz})^{22,23}$ It has been debated whether it is possible to record STN firing while patients receive general anesthesia and lose consciousness, as well as how these agents, such as intravenous propofol or inhaled desflurane, might dampen neuronal activity. The findings of our study suggest that performing meticulous monitoring while administering desflurane to implant DBS electrodes in patients with PD not only allows successful electrophysiological mapping but also avoids precluding a subset of patients who are not suitable for surgery under local anesthesia. ${ }^{8}$

The dorsolateral subterritory of the STN has long been considered an important part of the sensorimotor circuit of the basal ganglio-thalamo-cortical network and implicated as underpinning the pathophysiology of PD.,19 In accordance with clinical observations, high-frequency DBS within this region has also been demonstrated to achieve the best motor outcome for patients with PD and avoids the neuropsychological side effects due to current diffusion. ${ }^{24}$ Our findings demonstrate significant bursty firing and synchronization of oscillatory cells within the dorsolateral STN, which are similar to those of previous studies. Seifried et al.'s study not only showed the characteristic firing types of STN cells but also highlighted the importance of various percentages of firing patterns on the delineation of STN topography. ${ }^{22}$

After we applied MNS during STN recording, the percentage of STN oscillatory neurons increased significantly. Previous studies revealed a decreasing incidence of oscillatory neuronal activity within the STN after active limb movements, subthalamic stimulation, or levodopa administration, implying an interaction between motor cortex activation and the firing patterns of neurons. However, sensory input, such as MNS, has been shown to modulate the firing characteristics of the STN and induce very fast oscillations. ${ }^{7}$ Sensory stimulation could change motor cortex excitability, and a sensory-conditioned stimulus such as MNS could inhibit the motor cortex, which may indirectly lead to the increased oscillation of STN neurons. ${ }^{21}$

Additionally, MNS has been used as a reliable tool to identify basal ganglia structures from SSEP recordings and has been even more widely applied toward neural firing characterization and elucidation of the mechanisms of neural oscillation. Most of these reports chose local field potential (LFP) to identify the specific features of STN. ${ }^{12,18}$ In the other reports, using single neuronal discharges to facilitate delineation of STN in patients with PD has also been proven to be feasible intraoperatively or through offline analysis. ${ }^{17,23}$ Another study that analyzed the relationship between LFP and single neuronal discharges in STN showed that LFP illustrated synchronous activity of the neuronal population. ${ }^{13}$ Taking all of these data together, it is very difficult to decide which neuronal cacophony is better, but both LFP and single neuronal discharge play pivotal roles for us to treat disease with neurophysiological problems, such as PD.

Compared with STN firing without MNS, the mean firing frequencies of STN neurons, as well as the asymmetry index and coefficient of variance of the ISIs, remained similar during MNS. Nonetheless, our observation that the PSD of beta-band firing-which has been evaluated further during dorsolateral STN analysis-was remarkably augmented during MNS suggests that MNS can also enhance low-frequency oscillation in addition to ultra-fastfrequency oscillation, and facilitate single-unit recording of STN neurons during general anesthesia.

Although we can achieve recording of the STN under general anesthesia, we did encounter several difficulties that may explain why the mean firing rate was lower under desflurane general anesthesia. First, prolonged surgical duration may cause an accumulation of anesthetic concentration. In such circumstances, we need to lower the MAC of the patient and wait for a proper regain of signal. Second, a damaged MER electrode may hinder accurate neural signal recording. We need to check the impedance of the microelectrode regularly during MER. Lastly, we relied on the MAC to ensure proper sedation. However, we found a nonlinear correlation between the level of the MAC and characteristics of MER. Whether other physiological signatures could help us quantify the depth of anesthesia more accurately may need further research.

\section{Conclusions}

Analyzing the characteristics of MER within the dorsolateral STN during baseline and MNS confirmed the feasibility of differentiating STN firing while patients with PD undergo surgery under general anesthesia. MNS not only allows the enhancement of background oscillations and changes in spiking patterns but also may facilitate localization of the STN for patients with PD under general anesthesia and allow more candidates to undergo DBS operations.

\section{Acknowledgments}

We are grateful for the support from Master Cheng Yen, President of the Tzu-Chi Foundation, and would like to acknowledge Miss Tingwen Ho for data processing. 


\section{References}

1. Abosch A, Hutchison WD, Saint-Cyr JA, Dostrovsky JO, Lozano AM: Movement-related neurons of the subthalamic nucleus in patients with Parkinson disease. J Neurosurg 97:1167-1172, 2002

2. Amirnovin R, Williams ZM, Cosgrove GR, Eskandar EN: Visually guided movements suppress subthalamic oscillations in Parkinson's disease patients. J Neurosci 24:1130211306, 2004

3. Chan HL, Lin MA, Lee ST, Tsai YT, Chao PK, Wu T: Complex analysis of neuronal spike trains of deep brain nuclei in patients with Parkinson's disease. Brain Res Bull 81:534542,2010

4. Chen SY, Lee CC, Lin SH, Hsin YL, Lee TW, Yen PS, et al: Microelectrode recording can be a good adjunct in magnetic resonance image-directed subthalamic nucleus deep brain stimulation for parkinsonism. Surg Neurol 65:253-261, 2006

5. Chen SY, Tsai ST, Lin SH, Chen TY, Hung HY, Lee CW, et al: Subthalamic deep brain stimulation in Parkinson's disease under different anesthetic modalities: a comparative cohort study. Stereotact Funct Neurosurg 89:372-380, 2011

6. Cimenser A, Purdon PL, Pierce ET, Walsh JL, SalazarGomez AF, Harrell PG, et al: Tracking brain states under general anesthesia by using global coherence analysis. Proc Natl Acad Sci U S A 108:8832-8837, 2011

7. Hanajima R, Chen R, Ashby P, Lozano AM, Hutchison WD, Davis KD, et al: Very fast oscillations evoked by median nerve stimulation in the human thalamus and subthalamic nucleus. J Neurophysiol 92:3171-3182, 2004

8. Harries AM, Kausar J, Roberts SA, Mocroft AP, Hodson JA, Pall HS, et al: Deep brain stimulation of the subthalamic nucleus for advanced Parkinson disease using general anesthesia: long-term results. J Neurosurg 116:107-113, 2012

9. Harrison RR: A low-power intergrated circuit for adaptive detection of action potentials in noisy signals, in The 25th Annual International Conference of the IEEE Engineering in Medicine and Biology Society. New York: IEEE, 2003, Vol 4, pp 3325-3328

10. Hertel F, Züchner M, Weimar I, Gemmar P, Noll B, Bettag $\mathrm{M}$, et al: Implantation of electrodes for deep brain stimulation of the subthalamic nucleus in advanced Parkinson's disease with the aid of intraoperative microrecording under general anesthesia. Neurosurgery 59:E1138, 2006

11. Israel Z, Burchiel KJ: Microelectrode Recording in Movement Disorder Surgery. New York: Thieme, 2004

12. Klostermann F, Vesper J, Curio G: Identification of target areas for deep brain stimulation in human basal ganglia substructures based on median nerve sensory evoked potential criteria. J Neurol Neurosurg Psychiatry 74:1031-1035, 2003

13. Kühn AA, Trottenberg T, Kivi A, Kupsch A, Schneider GH, Brown P: The relationship between local field potential and neuronal discharge in the subthalamic nucleus of patients with Parkinson's disease. Exp Neurol 194:212-220, 2005

14. Lin SH, Chen TY, Lin SZ, Shyr MH, Chou YC, Hsieh WA, et al: Subthalamic deep brain stimulation after anesthetic inhalation in Parkinson disease: a preliminary study. J Neurosurg 109:238-244, 2008

15. Mallet L, Schüpbach M, N'Diaye K, Remy P, Bardinet E, Czernecki V, et al: Stimulation of subterritories of the subthalamic nucleus reveals its role in the integration of the emotional and motor aspects of behavior. Proc Natl Acad Sci U S A 104:10661-10666, 2007
16. Maltête D, Navarro S, Welter ML, Roche S, Bonnet AM, Houeto JL, et al: Subthalamic stimulation in Parkinson disease: with or without anesthesia? Arch Neurol 61:390-392, 2004

17. Moran A, Bergman H, Israel Z, Bar-Gad I: Subthalamic nucleus functional organization revealed by parkinsonian neuronal oscillations and synchrony. Brain 131:3395-3409, 2008

18. Pesenti A, Priori A, Locatelli M, Egidi M, Rampini P, Tamma F, et al: Subthalamic somatosensory evoked potentials in Parkinson's disease. Mov Disord 18:1341-1345, 2003

19. Romanelli P, Bronte-Stewart H, Heit G, Schaal DW, Esposito $\mathrm{V}$ : The functional organization of the sensorimotor region of the subthalamic nucleus. Stereotact Funct Neurosurg 82:222-229, 2004

20. Romanelli P, Heit G, Hill BC, Kraus A, Hastie T, BrontëStewart HM: Microelectrode recording revealing a somatotopic body map in the subthalamic nucleus in humans with Parkinson disease. J Neurosurg 100:611-618, 2004

21. Sailer A, Molnar GF, Paradiso G, Gunraj CA, Lang AE, Chen R: Short and long latency afferent inhibition in Parkinson's disease. Brain 126:1883-1894, 2003

22. Seifried C, Weise L, Hartmann R, Gasser T, Baudrexel S, Szelényi A, et al: Intraoperative microelectrode recording for the delineation of subthalamic nucleus topography in Parkinson's disease. Brain Stimulat 5:378-387, 2012

23. Toleikis JR, Metman LV, Pilitsis JG, Barborica A, Toleikis SC, Bakay RA: Effect of intraoperative subthalamic nucleus DBS on human single-unit activity in the ipsilateral and contralateral subthalamic nucleus. J Neurosurg 116:1134-1143, 2012

24. Tsai ST, Lin SH, Lin SZ, Chen JY, Lee CW, Chen SY: Neuropsychological effects after chronic subthalamic stimulation and the topography of the nucleus in Parkinson's disease. Neurosurgery 61:E1024--E1030, 2007

25. Weaver FM, Follett K, Stern M, Hur K, Harris C, Marks WJ Jr, et al: Bilateral deep brain stimulation vs best medical therapy for patients with advanced Parkinson disease: a randomized controlled trial. JAMA 301:63-73, 2009

26. Yamada K, Goto S, Kuratsu J, Matsuzaki K, Tamura T, Nagahiro S, et al: Stereotactic surgery for subthalamic nucleus stimulation under general anesthesia: a retrospective evaluation of Japanese patients with Parkinson's disease. Parkinsonism Relat Disord 13:101-107, 2007

27. Zaidel A, Spivak A, Grieb B, Bergman H, Israel Z: Subthalamic span of beta oscillations predicts deep brain stimulation efficacy for patients with Parkinson's disease. Brain 133:2007-2021, 2010

\section{Author Contributions}

Acquisition of data: Tsai. Analysis and interpretation of data: Tsai, Chuang, Kuo. Drafting the article: Tsai, Chuang. Critically revising the article: SY Chen. Reviewed submitted version of manuscript: SY Chen, Tsai, Kuo, Chao, Hung. Approved the final version of the manuscript on behalf of all authors: SY Chen. Administrative/technical/material support: YT Chen.

\section{Correspondence}

Shin-Yuan Chen, Department of Neurosurgery, Tzu Chi General Hospital, 707, Sec. 3, Chung-Yang Rd., Hualien 970, Taiwan. email:william.sychen@msa.hinet.net. 